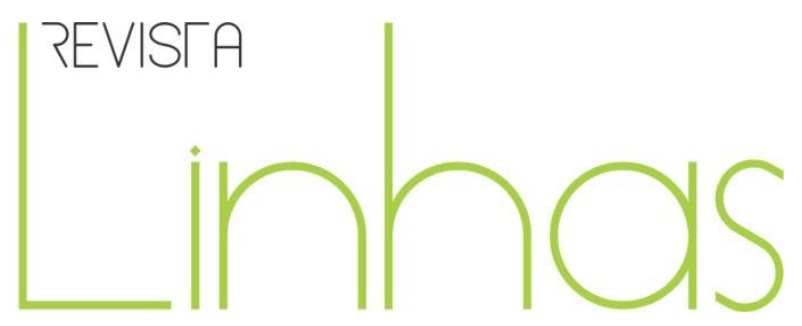

\title{
Le routine: da consuetudini sterili ad azioni fertili
}

\begin{abstract}
Riassunto
La giornata educativa dei bambini piccoli (0/6) è scandita da esperienze che si ripetono regolarmente nel tempo: lavarsi, mangiare, riposare... Chiamiamo questi momenti routine, perché si ripetono nel tempo e nel loro modo di svolgersi. Le routine possono essere vissute come consuetudini sterili, ma anche come azioni fertili, come delle semplici ripetizioni o come momenti di arricchimento e di cura. In questo secondo caso, con i bambini più grandi, occorre ripensare all'organizzazione delle routine perché esse siano - nella scuola dell'infanzia come al nido - una esperienza di arricchimento personale e di cittadinanza attiva.
\end{abstract}

Parole chiave: Quotidianità. Cura. Cittadinanza Attiva.

\author{
Gianfranco Staccioli \\ Università di Firenze - Itália \\ gianfranco.staccioli@unifi.it
}

\section{Para citar este artigo:}

STACCIOLI, Gianfranco. Le routine : da consuetudini sterili ad azioni fertili. Revista Linhas. Florianópolis, v. 19, n. 40, p. 74-93, maio/ago. 2018. 


\section{The routines: from dull As rotinas: de hábitos habits to fertile gestures \\ estéreis a ações férteis}

\begin{abstract}
The day in early childhood education is given structure by certain experiences which repeat themselves regulary: washing one's hands, eating, resting... One calls these moments "routine". They may be lived as dull habits, but also as fertile gestures, as mere ripetition but also as enriching occasions of caregiving. We need to think about how best to organize our "routines" in order that, in nursery schools as well as in creches, they may be lived as personal enrichment and as active citizenship.
\end{abstract}

Keywords: Daily Life. Care. Active Citizenship.

\section{Resumo}

A jornada educativa das crianças pequenas (de o a 6 anos) é caracterizada por experiências que se repetem regularmente ao longo do tempo: lavar-se, comer, descansar... Chamamos esses momentos de rotina porque se repetem no tempo e na sua maneira de se desenvolver. As rotinas podem ser vividas como hábitos estéreis, mas também como ações férteis, como repetições simples ou como momentos de enriquecimento e cuidado. Neste segundo caso, com crianças mais velhas, é necessário repensar a organização das rotinas para ser - na pré-escola como na creche - uma experiência de enriquecimento pessoal e cidadania ativa.

Palavras-chave: Cotidiano. Educação Infantil. Cuidado. Cidadania Ativa. 


\section{Ripetizioni di senso}

Le strade possibili che consentono la crescita sono tante. Ce ne sono alcune promettenti, altre faticose, altre che risultano indispensabili, altre ancora che ci appaiono piacevoli o frizzanti. Quando una persona trova delle strade che sono utili per lui e quando queste strade sono anche condivise da chi vive con lui, si dice comunemente che questa persona ha preso delle buone o delle cattive abitudini. Prendere buone o cattive abitudini è un fatto molto legato alla cultura di appartenenza: mangiare per terra può essere accettato, sopportato, negato, esaltato, a seconda del contesto. Così per gli altri atti che si ripetono nella vita quotidiana: lavarsi, dormire, riposarsi, andare in bagno... Pensare alle abitudini come a comportamenti buoni o cattivi, non ci aiuta. Forse sarebbe meglio pensare alle abitudini come a delle azioni fertili o a delle consuetudini sterili.

Le strade dei cammini abitudinari (in francese route significa strada, il termine routine deriva da lì) hanno una doppia valenza: da una parte nascono da spinte profonde, essenziali, necessarie. Di esse non si può fare a meno, non si può smettere di mangiare, di dormire o di svolgere altre funzioni legate al benessere corporeo e psichico. Ma, poiché le dobbiamo esercitare con continuità, esse corrono il rischio di diventare consuete, indispensabili, dei tran tran statici; esse tendono a diventare consuetudini e ripetizioni quasi automatiche. Le routine, nel pensiero comune, sono spesso considerate il dejà vu della vita. Sembra che non arricchiscano, che non aggiungano niente a ciò che già si conosce. Vanno compiute, eseguite, ripetute in un tempo strettamente necessario per svolgerle. In questo modo le abitudini risultano consuetudinarie, meccaniche, fino anche ad essere percepite come noiose. Allora le routine si trasformano in consuetudini sterili.

Noi sappiamo che per i bambini la cosiddetta routine non rappresenta una consuetudine sterile. Fra le due tendenze (quella ripetitiva e noiosa della consuetudine e quella di vitale legata alla scoperta del mondo) prevale quella che poggia sulla curiosità, che scopre un ripetersi sempre diverso, quella collegata alla meraviglia del particolare,

all'emozione del consueto non previsto, all'esplorazione del conosciuto. Così come avviene quando un bambino apre in bagno un rubinetto e l'acqua scende sulla sua mano, mostrandosi con infinite e imprevedibili forme. Le routine per i bambini sono più spesso 
delle azioni fertili, specialmente quando anche il contesto le riconosce e le valorizza. Per i bambini la ripetitività è anche sicurezza, è àncora per fermare il mondo agitato che scorre quotidianamente con loro, è quel rito evocato dalla volpe del Piccolo Principe che gli chiede di ritornare alla stessa ora perché deve sapere "a che ora devo prepararmi il cuore". Ripetitività si coniuga nell'infanzia a sicurezza e ricchezza, ed è allo stesso tempo sempre uguale e sempre diversa. Le routine rassicurano ed innovano, al tempo stesso.

Le routine dei bambini sono più spesso delle azioni fertili. Quelle degli adulti sono più frequentemente delle consuetudini sterili. Le routine dei bambini non si ripetono, perché in molte di esse i piccoli riescono a trovare sempre qualcosa di nuovo, di diverso, di inesplorato. In questo senso parlare di routine riferendosi ai bambini è un controsenso. Essendo le azioni del quotidiano sempre diverse per loro, mangiare, riposare, lavarsi non sono routine nel senso comune del termine. Noi adulti dobbiamo fare molti sforzi per rivedere il quotidiano ripetersi delle azioni consuete. Ed altrettanto sforzo dobbiamo farlo per far diventare fertili anche molte altre azioni quotidiane come le attività all'aperto, quelle in giardino o quelle che, nelle prime istituzioni educative, venivano chiamate "gioco libero". Come è facile capire, il gioco libero non esiste. Un bambino completamente spontaneo non esiste. Un bambino ed il suo gioco sono sempre condizionati, dal contesto, dalle mode, dalle condizioni culturali del momento. Quando bambini nella scuola dell'infanzia giocano da soli (anche se stanno uno accanto all'altro) con i loro smart phone, si può dire che stiano facendo un "gioco libero"? O stanno agendo fortemente direzionati da modelli economico culturali? Il gioco dei bambini, quello non diretto dagli adulti, rappresenta sempre il mondo dei grandi, con le sue abitudini, le mode, i valori, le attese che lo contraddistinguono. Una consuetudine fertile dedicata al tempo del gioco all'aria aperta, dovrebbe rientrare a pieno titolo nelle cosiddette routine.

La fondatezza dell'idea pedagogica di mettere in valore le routine nella fascia $0 / 3$ è nota. Da anni c'è una vasta letteratura al riguardo. I nidi italiani hanno sviluppato una particolare sensibilità al tema, soffermandosi sui singoli aspetti di ciascuna routine (vedi anche la bibliografia di riferimento). Questo tema è molto meno sentito nella fascia 3/6, ma ci sarebbe molto da dire anche riferendosi ai ragazzi più grandi (Staccioli, 1997). Le routine offrono ai bambini piccoli (o/3) un appiglio sicuro e favoriscono l'equilibrata 
organizzazione del tempo e dello spazio. Offrono una sistemazione coerente del prima e del dopo, consentono di prevedere cosa accadrà e di organizzarsi di conseguenza; sono esperienza comunitaria e comunicabile verso i pari e verso gli adulti; consentono un contatto concreto con bambini provenienti da culture diverse; favoriscono la narrazione e la condivisione... Le routine rappresentano anche il luogo di nuove acquisizioni conoscitive: "le routine, i rituali, le regole riassumono forme convenzionali di conoscenza che $\mathrm{i}$ bambini apprendono e in base alle quali riescono ad orientarsi culturalmente (Catarsi, Freschi, 2013, p. 26)

I rituali (a partire dall'ambientamento) favoriscono un processo di integrazione serena: entrare, spogliarsi, incontrare lo stesso adulto, accostarsi al altri bambini, salutare la mamma o il nonno, sono atti che consentono non solo una rassicurazione psichica, ma anche una stabilizzazione dello spazio temporale, relazionale e affettivo. Quando i bambini sono messi in condizione di comprendere come si svolge la giornata al nido - e qui entra in gioco anche il tema del ritmo, della lentezza, della calma - diventano più responsabili, più accorti, più autonomi. Non c'è da soffermarsi molto sulle caratteristiche educative delle routine. I nidi in Italia e in Europa ne hanno fatto un emblema ed un segno di riconoscimento pedagogico. Semmai ci sarebbe da pensare a come questi segnali vengono (o possono venire) utilizzati e sviluppati nella scuola dell'infanzia. E qui il discorso si allarga...

La scuola dell'infanzia italiana ha conquistato - fino dagli anni Settanta (Pescioli, 1972) - il diritto di essere considerata un "prima scuola". Un diritto che in questi anni è diventato comune a molti, ed ha consentito di coprire con la scolarizzazione oltre il 90\% dei bambini. Un diritto che si è vestito di proposte didattiche non casuali, organizzate, guidate (espresse anche dagli Orientamenti emanati in Italia a livello nazionale nel 2012). Un diritto che ha cercato la continuità con l'ordine di scuola successivo (la scuola primaria, che in Italia inizia a sei anni). A cinque anni, in tutte le classi della scuola dell'infanzia, si pensa alla scrittura, alla lettura, al calcolo. In forme discrete o in maniera schematica (con specifiche schede di apprendimento), oppure seguendo approcci legati alla costruzione delle conoscenze. Ogni insegnante è certo che ciò che verrà appreso dai bambini nella scuola dell'infanzia, sarà sviluppato nella scuola primaria, dalle insegnanti che verranno. 
Se questo ragionamento - che risulta un procedimento corretto per quanto riguarda la continuità scolastica degli apprendimenti - lo applicassimo anche alle dimensioni relazionali, affettive e cognitive, allora anche le routine (così come sono state sperimentate nel nido) potrebbero acquistare un significato diverso nella scuola dell'infanzia. Come si sviluppano nella scuola dell'infanzia le routine? Si può dire che durante il periodo $0 / 3$ anni siano già state raggiunte tutte le competenze all'autonomia del vivere quotidiano? Si può affermare che le attenzioni dei bambini ed il loro interesse per la vita quotidiana (con le loro possibili scoperte) siano scomparse? Cosa comporta una maggiore attenzione alle routine, da parte delle insegnanti? Come si fa - ad esempio - ad organizzare il pranzo o la pratica dell'andare in bagno in continuità con le conoscenze e competenze acquisite al nido? Come organizzare spazi, tempi, relazioni, perché vi sia un costante arricchimento che produca quelle azioni fertili alle quali si è accennato? Ripercorreremo brevemente, qui di seguito, alcuni momenti di routine come il pranzo, la cura del corpo, il riposo, aggiungendo una routine inconsueta (gli spazi esterni) che secondo noi rappresenta una presenza quotidiana non sempre valorizzata ed utilizzata.

\section{Mangiare a scuola}

Il pranzo si presta facilmente ad una riflessione sulla continuità. Al nido la successione delle modalità organizzative e sociali per acquisire competenze diverse sono molto chiare. Nei tre anni ipotetici che un bambino può passare al nido, anche la disposizione materiale cambia continuamente (in braccio, sul seggiolone, usando la sedia con braccioli, su una piccola sedia, con una sedia più grande...), così come cambia l'altezza dei tavoli, cambia il modo di mangiare (un po' imboccati e un po' da soli, con le mani e con le posate, con il coltello a punta tonda,,,), cambiano le relazioni interpersonali (con l'adulto di riferimento, con pochi compagni, con un piccolo gruppo), cambia il contributo che viene chiesto ai bambini (prima vengono serviti, poi possono servirsi da soli, poi aiutano a mettere in tavola le posate, le stoviglie, imparano ad apparecchiare, a sparecchiare, contribuiscono a riordinare le stoviglie sporche... ). Man mano che i bambini 
crescono, cambiano le regole, cambia la disposizione a tavola, cambiano le relazioni ... (AA.VV, 2013).

Con gli stessi principi di cambiamento e di sviluppo si dovrebbe organizzare il pranzo nella scuola dell'infanzia. Se la dimensione amicale e relazionale si intensifica, occorrerà organizzarsi perché sia possibile per i bambini parlare con calma con i compagni e occorrerà che ci sia anche una maggiore autonomia nella gestione del tempo tavola. Se la competenza linguistica va accrescendosi, occorrerà sostenere gli scambi verbali, favorendo l'intimità relazionale fra persone che si conoscono e che mangiano assieme allo stesso tavolo. Se la dimensione di responsabilizzazione va accresciuta, anche i compiti dei bambini perché funzioni bene il pranzo, dovranno cambiare e risultare più articolati e autoresponsabilizzanti. Se la dimensione culturale si fa più complessa, ci sarà molto da fare per riprendere, valorizzare, ampliare le "chiacchiere" attorno al cibo, alla sua provenienza, alla sua qualità ecologica, ai suoi costi economici, ai suoi effetti sulla crescita ed il benessere personale.

Le possibilità organizzative sono molte e molte ed anche diverse fra loro sono state sperimentate. Ci sono scuole dell'infanzia dove si adotta una distribuzione degli alimenti stile mensa (un adulto che mette il cibo nei piatti dei bambini). Ci sono scuole dove si è scelto di usare la forma del self service ed ogni bambino passa davanti agli addetti alla distribuzione, prende la porzione che gli spetta e va a sedersi ad un tavolo. $\mathrm{Ci}$ sono scuole dove il cibo viene sistemato in vassoi che contengono la quantità adatta per un piccolo gruppo ed il cibo viene distribuito al tavolo da un bambino del gruppo. Ci sono scuole dove gli adulti non mangiano con i bambini, o mangiano in un tavolo separato, o mangiano con un gruppo di bambini. In certi casi i bambini mangiano con uno stesso gruppo, in altri si siedono liberamente, in altri ancora stanno insieme in lunghe tavolate... Si tratta di una gamma infinita di possibilità e di variazioni che vanno pensate e calibrate perché la scelta dell'una o dell'altra non ha lo stesso valore e non manda gli stessi messaggi. C'è sempre da chiedersi (ed in questo sta la professionalità delle educatrici e degli educatori) quale sia la forma migliore e quali siano le modalità organizzative più efficaci perché si possa sviluppare una crescita continua dei bambini, sia sul piano 
dell'autonomia (responsabilità sociale), sia sul piano relazionale (consapevolezza affettiva), sia sul piano cognitivo (consapevolezza comunicativa e riflessiva).

Si sarà notato come in queste brevi note inerenti al pranzo non si sia fatto cenno all'aspetto nutrizionale. Anche cosa si mangia è importante, come è importante cucinare in modo che le cose da mangiare siano buone e ben presentate. L'atto di nutrirsi (o con i piccolissimi, di essere nutrito) non riguarda solo gli aspetti fisiologici e di appetito. Si mangia meglio quando il contesto fa star bene, quando c'è sintonizzazione emotiva e relazionale con gli adulti e con i compagni. Il cibo, oltre che di calorie, è un grande veicolo di vibrazioni che nutre il corpo di emozioni e di affetti. L'arte di far mangiare è propria di quegli adulti che sono consapevoli di quanto intreccio ci sia fra cibo e psiche, fra fame fisica e relazione con gli altri, fra abitudini consuetudinarie ed alimenti non conosciuti. II momento del mangiare diventa così una azione quotidiana fertile e piacevole, sia per il bambino che per l'adulto che è con lui e con i compagni che stanno a tavola con lui. E man mano che i bambini crescono, il momento del pasto diventa palestra di esercizio di una autonomia sostenuta dal piacere.

Qualità, estetica e relazioni favoriscono il piacere del mangiare. Lo spazio dove si mangia, come lo si arreda, come vengono raggruppati i bambini, come vengono sistemate le brocche dell'acqua o gli stracci per eventuali necessità... sono tutti elementi che condizionano le relazioni ed il benessere conviviale. "Lo spazio esprime valori, pensieri, ha un suo linguaggio silenzioso, ma potente" (Galardini, 2003, p. 49). L'organizzazione spaziale comprende anche la dimensione degli arredi, quella delle posate, il tipo di tovaglia da mettere, le modalità di scelta del tavolo dove sedersi, la predisposizione delle condizioni per una sparecchiatura collaborativa, la disposizione delle educatrici e del personale. Ci sono certamente ragioni nutrizionali da tenere presenti, come ci sono ragioni economiche che si intrecciano con il mangiare a scuola (in Italia si predilige cibi a "chilometro zero", cioè prodotti che sono sani e con coltivazione biologica). Altrettanto importanti sono le condizioni di lavoro delle persone che si occupano del cibo: un clima tranquillo in cucina e delle buone relazioni fra inservienti ed educatori, rendono il pranzo più gradevole e funzionale. La dimensione educativa non si 
ferma alla organizzazione degli spazi, delle relazioni adulto/bambino, ma deve toccare l’organizzazione della scuola ed il suo rapporto con la società più ampia.

\section{La cura del corpo}

Uno stesso ragionamento si può fare anche per i momenti dell'andare in bagno: accompagnati, da soli, utilizzando e curando le cose personali e quelle comuni, favorendo esperimenti in piccolo gruppo autonomo al lavandino, organizzando supervisioni fatte dai bambini perché tutto sia sempre in ordine e a disposizione, favorendo piccole esperienze scientifiche con l'acqua, riflettendo su come fa l'acqua ad arrivare a scuola.... Al nido i bambini piccoli vengono sostenuti e incoraggiati perché possano lentamente divenire autonomi. Il rapporto adulto/bambino passa dalla relazione duale al fasciatoio, ad un rapporto più indipendente verso le necessità corporee (dalla capacità a riconoscere quando è il momento giusto per fare i propri bisogni, alla capacità di usare le scalette per arrivare al fasciatoio, allo spostarsi per andare al gabinetto...). Le funzioni che con i piccolissimi competono all'adulto (questa routine pluriquotidiana viene spesso denominata "il cambio") successivamente passano mano a mano ai bambini: salire la scaletta del fasciatoio, andare in bagno accompagnati o da soli, usare la carta igienica, lavarsi con il sapone, aprire e chiudere il rubinetto dell'acqua, asciugarsi... Si tratta di una infinità di conoscenze agite che sono indispensabili per garantire il benessere fisico, ma anche la serenità del corpo stesso. I momenti del cambio, del bagno, della cura fisica del corpo sono momenti delicati anche perché è in questi momenti/luoghi che si pongono le basi per un approccio corretto alla scoperta delle specificità corporee e della sessualità. Sono anche momenti nei quali si manifesta il dualismo sporco/pulito che affascina e intimorisce ogni bambino: il messaggio dell'adulto che dice: "non sporcarti, la cacca è sporca, non toccare ..." si contrappone al bisogno di scoprire, manipolare, esplorare, conoscere.

I bambini piccoli, all'inizio imitano l'adulto, poi lo accompagnano nelle azioni di cura di sé, poi riescono a fare da soli. "Aiutami a fare da solo" è il conosciuto slogan montessoriano che è applicabile anche in questo caso. Ci sono un'infinità di azioni che 
sono necessarie nei momenti della cura del corpo e che richiedono la stessa attenzione che viene data al pranzo o ad altre routine. Quando i bambini sono più grandi, se hanno avuto una buona esperienza al nido, possono fare da soli molte cose. La cura e la predisposizione degli ambienti, dei tempi, dei materiali e gli strumenti relativi alle cure personali, non dovrebbero venir meno nella scuola dell'infanzia perché l'andare in bagno può rappresentare un quotidiano esercizio di responsabilità sociale. Non sprecare l'acqua, riporre l'asciugamano personale nell'apposito contenitore, lasciare pulito il bagno, tenere in ordine gli oggetti comuni o il sacchetto dell'igiene personale, mantenere ben sistemate le cose nel proprio armadietto, salvaguardare le attrezzature e gli strumenti presenti nell'ambiente, sono tutti comportamenti che richiedono maturazione e consapevolezza.

E' anche nell'uso del bagno che prendono vita le esigenze strettamente personali e quelle comunitarie. Il bagno va condiviso, come si condividono i luoghi e le cose della vita quotidiana in famiglia o nella società. Sporcare i muri, lasciare cartacce per terra, abbandonare bottigliette e tanti altri comportamenti che dimostrano disaffezione per le cose di tutti, forse non hanno una diretta conseguenza con le esperienze infantili relative al bagno, ma certamente si riferiscono all'avere cura di sé e degli altri, di avere attenzione per il personale ed il comunitario, hanno a che vedere con il rispetto degli altri e con la responsabilità personale. Tutte cose che possono essere azioni fertili con i bambini nella prima scuola, quando la loro vita quotidiana viene attraversata - per usare una locuzione non solo italiana - da un'esperienza di cittadinanza attiva.

Siamo, come si vede, molto lontani dall'accezione "medica" di un corpo che deve essere protetto perché non si ammali o perché non si faccia male (due aspetti certamente da non trascurare). La cura del corpo si riferisce piuttosto al fatto che si deve imparare (adulti e bambini) a porre attenzione a stessi e agli altri, ad avere sollecitudine per i propri e gli altrui bisogni, a vivere la motricità con piacere senza sforzo competitivo. Il corpo curato nei confronti di se stesso deve imparare a conoscersi, e la conoscenza del corpo (fisico e psichico) è un percorso che non ha termine in tutta la vita. Il corpo di ogni bambino, va curato anche nei confronti degli altri, perché possa sviluppare le proprie potenzialità collaborative piuttosto che quelle antagoniste o aggressive. Per far questo non c'è da guarire un corpo, casomai da c'è accompagnare quei corpi che - per spinte 
proprie della cultura di appartenenza - tendono a sciuparsi, ad infiacchirsi o a richiedere troppo a se stessi. Sono tendenze che appaiono sempre più frequentemente man mano che i bambini crescono e che richiedono, da parte degli adulti, un intervento intenzionale e consapevole nelle pratiche quotidiane di cura.

Un discorso simile si potrebbe fare per altri momenti della vita quotidiana. Le routine nel loro insieme - anche da un punto di vista della quantità di tempo che occupano nel nido o nella scuola dell'infanzia - rappresentano la dimensione più ampia del vivere a scuola. Possono essere vissute come noiose o semplicemente come azioni futili da compiere. O possono diventare una palestra di cittadinanza. Nessun altro insegnamento viene praticato con la stessa quantità di tempo che, nel loro insieme, rivestono le routine. Sono questi momenti personali e al tempo stesso sociali, che scandiscono il passare delle ore e dei giorni, che creano abitudini, che modellano comportamenti, che sostengono la consapevolezza che l'individualità e la socialità sono due poli che possono e devono convivere integrandosi a vicenda. In una scuola fiorentina del secondo dopoguerra (Scuola Città Pestalozzi) si insegnava, attraverso l'esperienza quotidiana, a divenire "cittadini liberi”. I ragazzi fino dalle prime classi, assumevano compiti e responsabilità (con l'occhio vigile degli operatori scolastici) che normalmente venivano demandate agli adulti (dall'accoglienza al portone scolastico, all'aiuto in cucina, alla cura del giardino, alla valutazione dei comportamenti inadeguati dei compagni). Si era cittadini fino da piccoli, a Scuola Città, ma per divenire "cittadini liberi" occorreva un esercizio quotidiano, che poteva durare anni. La democrazia, la libertà, i diritti ed i doveri stanno nel loro esercizio quotidiano. Le routine rappresentano un campo privilegiato per l'educazione sociale e per una cittadinanza attiva.

\section{II riposo}

"I bambini non vengono a scuola per dormire", ha detto una dirigente scolastica ad una mamma che chiedeva perché il suo bambino, che al nido riposava al pomeriggio, non poteva più farlo nella scuola dell'infanzia. L'opinione di questa dirigente è ancora molto diffusa. Molti insegnanti e tanti genitori pensano ancora che la scuola sia fatta per 
lavorare, per imparare, per studiare. II tempo del sonno appare per loro sprecato, perché sembra non aggiunga nulla all'apprendimento, anzi che tolga spazio ad altre attività (laboratoriali o specifiche) che vengono considerate più importanti. II sonno sarebbe in fondo un vuoto di programma, una pausa improduttiva. Tanto varrebbe ridurlo o abolirlo del tutto. A queste considerazioni negative, rispetto al riposo dei bambini, se ne aggiungono altre di carattere più organizzativo: le scuole dell'infanzia non sono adeguatamente attrezzate per avere delle "stanze del sonno" o degli ambienti specificatamente pensati per restare in silenzio leggendo o stando in silenzio.

Nell'affermare che i bambini della scuola dell'infanzia hanno bisogno di riposo, si va un po' controcorrente. Anche perché il riposo non viene considerato in maniera "educativa" neppure nelle famiglie. Spesso i bambini lo vivono in modo sregolato, vanno a letto tardi, si addormentano con lo smart fone in mano, rimangono alzati finché non crollano o fino che non vengono trascinati dal sonno. Molti genitori si lamentano che i bambini con loro sono irrequieti, nervosi e instabili. Le insegnanti confermano che questo comportamento avviene anche a scuola. I bambini passano velocemente da un interesse ad un altro, sono instabili, sono nervosi con i compagni. O, all'inverso, sono lenti, fiacchi, si addormentano sul banco. Ma sappiamo che si tratta di un circolo vizioso: più i bambini sono stanchi è più diventano agitati, più sono agitati, meno riescono a riposarsi. Fino a che non crollano, dovunque si trovino. E sappiamo anche che esiste una correlazione fra stanchezza e apprendimento. Quando si è sereni si impara di più e meglio. La velocità delle informazioni che riceviamo ogni giorno, non producono velocità di apprendimento. Anzi, lasciano che il pensiero fugga da se stesso. Per invertire la tendenza alla fretta (a casa come a scuola), occorrerebbe essere fortemente convinti che la calma, la Slow School (Ritscher, 2015), il ritmo lento (Zavalloni, 2009), rappresentano un valore perduto che va recuperato per vivere meglio (Honoré, 2014). La scuola può contribuire al benessere e alla formazione di una solida cultura personale, proprio accettando la regola del fare meno per riflettere di più. Se si fosse convinti di questo non sarebbe impossibile con-vincere anche le famiglie ed aiutarle a rallentare anche con i loro bambini.

Il riposo dei bambini fino a tre anni, è normale che venga predisposto con una attenzione al tempo, agli spazi, alle condizioni relazionali, perché il loro bisogno 
fisiologico è molto più evidente e non può essere sorvolato. I piccoli si addormentano dovunque, nel momento in cui ne sentono la necessità. I più grandi possono "trattenere" il riposo, ma con la conseguenza di diventare più eccitati e inquieti. Se si accogliesse l'ottica della lentezza, si dovrebbe ripensare l'organizzazione della la scuola dell'infanzia così da consentire momenti di riposo anche ai bambini di cinque anni. Può darsi che i più grandi non dormano, ma è importante prevedere almeno un'occasione quotidiana programmata per distendersi, per stare in silenzio, per stare con se stessi. La stanza del sonno può diventare la stanza del silenzio, la stanza dell'intimità, la stanza della tranquillità.

Stare a scuola comporta fatica, anche da un punto di vista acustico e relazionale. C'è sempre rumore, c'è sempre convivenza, ci sono stimoli continui. Il silenzio e la calma sono altrettanto importanti, e non trovano oggi molto spazio. Maria Montessori aveva “inventato" la "lezione del silenzio" ed annotava che “i bambini prendono molto interesse al silenzio; sembra che essi provino una specie di incanto; si potrebbe dire che sono rapiti in meditazione" (Montessori, 1970, p.49). Queste parole sono state redatte un secolo fa, per bambini che non conoscevano i ritmi frenetici e le stimolazioni tecnologiche ed ambientali dei bambini di oggi. Oggi fanno riflettere ancora di più, anche per quella parole "meditazione" usata dalla Montessori, una parola che, se considerata in senso laico, ci rimanda all'importanza della calma, di momenti intimi di riflessione e di pensiero, di silenzio esteriore e interiore.

"Il riposo è un rito rassicurante, da vivere con piacere, non deve essere un'imposizione forzata e leggermente punitiva. Nonostante alcuni problemi logistici reali (come ad esempio la mancanza di un ambiente ad hoc), bisogna evitare che il luogo del riposo diventi un "campo di sfollati”. Al riposo va data dignità, curando in dettaglio tutti gli aspetti pratici (l'illuminazione, la disposizione degli arredi, i tempi...). Ogni bambino dovrebbe avere una borsa personale con il necessario per il riposo (guancialino, copertina e lenzuolo oppure un leggero sacco a pelo facilmente lavabile). Le borse dovrebbero essere sistemate in un posto fisso, accessibile ai bambini..." (Ritscher, Staccioli, 2005, p. 90). Per avere continuità nido-materna occorre riconsiderare il riposo e la sua importanza proprio nel nostro tempo frettoloso. Occorre dare a questi momenti quella dignità 
pedagogica che essi meritano (come avviene nel nido). Sono momenti che vanno pensati anche nei dettagli perché anche la cura delle cose e degli ambienti sono elementi indispensabili per dare senso al riposo, al nido come nella scuola dell'infanzia.

\section{Gli spazi esterni}

L'uso quotidiano degli spazi esterni è un altro tema legato alle routine. Qui il discorso si fa più complesso, non tanto per ragioni pedagogiche, quanto per abitudini culturali che attraversano la scuola e le famiglie. II nido o la scuola vengono spesso identificati con l'edificio scolastico. Le mura proteggono, isolano, consentono raccoglimento, comunanza, controllo... Sapere che il proprio bambino sta in classe, sotto lo sguardo vigile di un adulto tranquillizza ogni genitore. E rende sereno anche ogni educatore che riesce, in un contesto controllato, a "tenere a bada" la situazione di un gruppo di bambini. Il controllo del gruppo o della classe è importante perché se i bambini rimangono abbandonati a se stessi, rischiano di fare la fine dei ragazzi de Il signore delle mosche di William Golding (1980). Golding, in reazione a molta letteratura dell'infanzia degli anni Cinquanta del secolo scorso (che rappresentava i bambini sempre buoni, giudiziosi, innocenti, allegri, pieni di buon senso), racconta una storia dove i ragazzi diventano "cattivi, crudeli, viziosi, sporchi e incapaci di vivere insieme" (p. 6). Golding ci dice che il contesto è determinante per i comportamenti di un gruppo.

La costruzione di un contesto sicuro e stimolante è importante nella organizzazione degli spazi esterni. L'esperienza e la vita di un bambino (o di un adulto) non è completa se la sua formazione scolastica si svolge sempre in classe o nei laboratori, in condizioni di costante "controllo" comportamentale e cognitivo da parte degli adulti. Un bambino che cresce ha bisogno anche di spazi dove ci possa essere una certa autonomia, anche di movimento e di raggruppamento. Nei nidi italiani ancora oggi si esce poco, nelle scuole dell'infanzia si esce quasi esclusivamente per la ricreazione. Lo spazio esterno è una routine da riconsiderare e riorganizzare. E' vero che negli ultimi anni si sta sviluppando un interesse maggiore per questo tema (come nell'Outdoor Education o 
nell'Educazione Attiva all'aria aperta), ma di strada da percorrere ce n'è molta (Ritscher, Staccioli, 2005).

Anche nelle attività all'aperto ci sono esigenze di gestione del contesto, che vanno gestite. con la stessa cura delle routine "classiche". Le regole sono le stesse: favorire le attività di piccolo o di piccolissimo gruppo, predisporre l'ambiente perché ci siano i materiali o gli stimoli necessari (come quando si pensa agli oggetti, alle condizioni, alle relazioni al momento del pranzo o del sonno). Fare in modo che la gestione dei piccoli progetti o delle iniziative porti ad una responsabilità prevalente dei bambini. Curare che queste iniziative consentano il "rischio", ma non determinino pericolo. Questo vuol dire che anche la posizione dell'adulto va ripensata e ricalibrata.

C'è un elemento particolare, relativo alle attività all'aria aperta, sul quale c'è da riflettere. Assieme alla preoccupazione di "perdere il controllo" dei bambini, ci sono resistenze implicite o esplicite che spesso accomunano genitori ed educatori: fuori fa freddo o piove e quindi la salute fisica sembra in pericolo (e allora non si esce); i bambini possono farsi male con legnetti o con gli alberi (e allora gli alberivengono eliminati o fasciati di gommapiuma per evitare incidenti); le sabbiere e comunque i giochi con la terra sono antigienici e portano a malattie (e allora il giardino viene trasformato in uno spazio completamente lastricato); piante, fiori o cespugli possono essere velenosi (e allora vengono inseriti prati artificiali). Queste resistenze sono anche comprensibili, ma piuttosto che agire per rimuoverle e per condizionare lo spazio, vengono talvolta usate come limiti invalicabili. L'esterno non è un campo minato. L'esterno può diventare una routine affascinante se si fanno i conti con questi aspetti e se si riescono a gestire in modo da far risaltare le valenze positive e specifiche che abbiamo ricordato essere presenti nelle routine tradizionali.

Rendere quotidiano il tempo educativo all'aria aperta è un passo molto grande. Uscire tutto l'anno, anche quando le condizioni atmosferiche o la temperatura non sono nella norma, è un passo ancora più grande. Uscire e considerare lo spazio all'aperto come un luogo che va curato come le altre routine è un altro salto di qualità che ancora non è stato assimilato e compreso dalle famiglie o dalle educatrici. L'organizzazione degli spazi esterni delle scuole infantili risponde molto spesso ad una logica di sfogo (uno spazio 
aperto e vuoto per correre, in modo da scaricare le energie compresse all'interno dell'aula) o di tempo libero (con attrezzature che ricordano i giardini pubblici o i parchi di divertimento, per alleggerire la fatica del lavoro “serio"). Un giardino che diventa routine ricco di azioni fertili, trasforma lo stare all'aperto in un "ambiente di apprendimento", fonte di stimoli e di possibilità: "ciò che un bambino può imparare stando fuori, per esempio, nel giardino della scuola, giocando, esplorando, reagendo alle sollecitazioni sensoriali e alle stimolazioni che riceve, costituisce il più formidabile ambiente di apprendimento di cui l'infanzia possa disporre, irriducibile a qualunque 'ambiente di apprendimento' interno, per quanto ben organizzato e gestito" (Farné, 2014, p. 257).

Inserire gli spazi esterni, così intesi, nel cesto educativo delle routine, facilita da una parte la valorizzazione di quelle tradizionali (anche mangiare, dormire ecc., possono essere predisposte ed utilizzate come “spazi di apprendimento”) e dall'altra facilita il superamento delle resistenze (legate alla salute fisica o alla sicurezza) che sono ancora molto diffuse, ancora in molti adulti.

\section{Le routine informali}

Le routine quotidiane sono, a nostro avviso, sia quelle tradizionalmente classificate come tali, sia quelle che possono diventarlo (come l'uscire quotidiano dalla classe), sia quelle che non hanno una forma prestabilita, ma che si ripetono ogni giorno, più volte. Quest'ultime andrebbero chiamarle "routine informali", perché sono più immateriali delle altre, ma richiedono anch'esse predisposizione, atteggiamento di ascolto, sostegno all’autonomia, intervento di aiuto, né più né meno delle altre routine.

In ogni momento della giornata educativa possono accadere degli imprevisti. Come sappiamo tutti, la vita non è completamente programmabile. Come già insegnava Cusano (De ludus globi, 1463) ai suoi studenti di filosofia, si può lanciare una palla tagliata a metà pensando che essa vada diritta, ma la palla, come le azioni che agiamo nella vita, sarà soggetta alla contingenza, alla fortuna e al caso Il percorso della palla è paragonabile al corso della vita (piena di imprevisti e di accidenti), così come nelle programmazioni scolastiche, lanciate come una palla tagliata a metà, non è detto che producano il 
previsto. Anzi, molto spesso è l'imprevisto che spunta, e questo suo quasi inarrestabile ripetersi, lo fa avvicinare, per certi versi alle routine. Farsene carico (che ovviamente non vuol dire lasciarsi trascinare dalla casualità e dall'inatteso) è una modalità di insegnamento che abbandona le programmazioni rigide e verificabili. L'inatteso è quella forza che (come ci ricorda Euripide) che viene sospinta da una forza sovrumana ("l'atteso non si compie e all'inatteso un dio apre la via"). Come dire, l'inatteso non solo non può essere evitato, ma dovrebbe essere sostenuto. Anziché attendersi l'atteso dai bambini che quotidianamente vivono nella scuola, occorrerebbe predisporsi all'inatteso. E, se del caso, servirsene ed utilizzarlo.

Luca e Gianni hanno cinque anni, sono in giardino, vicino ad un tiglio. Il tiglio proietta la propria ombra parte sul terreno, parte sul muro della scuola. Ad un certo punto fanno una scoperta inattesa. "Guarda maestra, guarda, ora l'ombra non c'è più." Gianni si è messo dapprima in modo da avere l'ombra proiettata sul muro e poi, con un balzo è saltato nella zona dell'ombra proiettata dal tiglio. "La mia ombra se l'è mangiata l'ombra grande. Prova anche te. Mangia anche la tua, vedrai", dice. Luca che ha assistito a tutta la scena domanda: “Maestra, perché quell’ombra si mangia le nostre ombre?”. Come rispondere ad una domanda del genere? Certo non con una disquisizione scientifica.

Il "gioco dell'inatteso" potrebbe continuare per esempio "meravigliandosi" del fatto che non sempre uno più uno, fanno due. Nel caso delle due ombre, uno più uno fa ancora uno. Un'ombra su un'altra ombra dà solo un'ombra. Oppure “meravigliandosi” del fatto che un oggetto piccolo possa fare un'ombra più grande di un oggetto ancora più grande, o che un'ombra può essere modellata e far apparire personaggi o animali... L'inatteso, l'informale, l'occasionale, sono parte del quotidiano, tornano continuamente, sempre diversi, e richiedono uno stesso atteggiamento, situazione per situazione, giorno per giorno, come fossero una routine. Come il mangiare, il dormire, il giocare, il curare il proprio corpo, la ripetizione della meraviglia (ripetere l'irripetibile è una bella contraddizione) è un tratto essenziale del vivere, un atteggiamento, 'un'azione fertile che fa da accompagnamento al vivere quotidiano. 
Valorizzare le routine, facendole entrare a pieno titolo nel curricolo di crescita ha anche lo scopo di consentire ai bambini di vivere la scuola, non solo di stare nella scuola. Non è facile realizzare una scuola dove i bambini stiano bene perché sentono che c'è vita, in ogni momento. C'è vita quando si intersecano le esigenze, i desideri, le storie personali, quelle dei bambini e quelle legate alla conoscenza di come va il mondo, di come il mondo era prima, di come forse sarà domani. I bambini hanno una curiosità infinita per la conoscenza, e tanto più quando si percorrono strade che risultano significative anche per loro.

Le attività di routine finora accennate rivalutano l'importanza di compiere esperienze motivanti, personalizzate, che consentono il piacere dell'esplorazione ed il potenziamento della curiosità. Attività che danno la possibilità ai bambini di crescere in autonomia, di mettersi alla prova di fronte a situazioni protette, di affrontare dimensioni culturali diverse, di sperimentare le proprie capacità personali, di guardare anche oltre le proposte dirette e preordinate dagli adulti.

Vivere la scuola e non nella scuola, significa anche percepire (da parte dei bambini, ma anche da parte degli adulti) che non ci sono attività di serie $A$ e attività di serie $B$. Che non ci sono momenti di lavoro e momenti di svago. Che non esiste un tempo libero, se non quello che viene liberato dalle scorie dei pregiudizi e dalle consuetudini non riflettute. Che si impara facendo e sperimentando, sostenuti dalla passione e dal piacere emozionato. Che si cresce solo misurandosi con il possibile e con il rischioso. Che si sta meglio quando si può agire e parlare in autonomia, in coppia o in piccolo gruppo. Che si sta bene quando gli adulti danno sicurezza senza essere opprimenti, quando conducono alla autonomia senza cedere all'abbandono, quando fanno sentire la loro vicinanza senza imporre i loro progetti.

Vivere la scuola è anche un grande esercizio di democrazia. Prendersi la responsabilità dei propri atti, confrontarsi con decisioni che non offrono certezza, condividere con grandi o piccoli aspirazioni e curiosità, educarsi al "senso di realtà" è il modo migliore per imparare a valutare e decidere con più consapevolezza. Queste e altre ancora sono le regole per quella che viene chiamata oggi cittadinanza attiva. Cittadini consapevoli che diventano "liberi”, perché fino da piccolissimi nell'agire quotidiano e 
routinario, hanno imparato a confrontarsi con se stessi, con gli altri e con quel complicato mondo degli adulti e le sue regole. Le routine non sono una incombenza solo necessaria, ma offrono la possibilità di rendere interessante ed arricchente la vita di tutti i giorni. Per l'oggi e per il domani.

\section{Riferimenti}

AA.VV., A tavola si cresce, <http://www.mediatecatoscana.net/sociale.php>, 2013.

Boffo Vanna, La cura in pedagogia, Clueb, Bologna, 2006.

Bortolotti Alessandro, Schenetti Michela, Outdoor education: l'educazione attiva all'aperto, numero speciale di Bambini, n.4/5, Spaggiari Editori, Parma, 2015.

Catarsi Enzo (a cura di), Il sonno nel nido d'infanzia, Junior, Azzano San Paolo (Bergamo), 2008.

Catarsi Enzo, Freschi Enrica, Le attività di cura nel nido d'infanzia, Junior, Azzano San Paolo Bergamo, 2013.

Farné Roberto, Agostini Francesca. (a cura di), Outdoor Education., Edizioni Junior, Parma, 2014.

Galardini Anna Lia, Crescere al nido. Gli spazi, i tempi, le attività, le relazioni, Carocci, Roma 2003.

Golding William, II signore delle mosche, Mondadori, Milano, 1980 (ed. or. Lord of the Files, 1954).

Goldshmied Elinor, Jackson Sonia, (1996) Persone da zero a tre anni. Crescere e lavorare nell'ambiente del nido, Junior, Bergamo (ed. or. People under Tree. Young Children in Day Care, Routledge, London,1994). 
Honoré Carl, Elogio alla lentezza. Rallentare per vivere meglio, BUR, Milano 2014, (ed. or. Praise of Slowness, 2004).

Honoré Carl, The Beauty of Slowing Down, ebook, 48 pages Published February 7th 2012 by Harper One.

Marchioli Giulia, Vigoni Simona, Vita quotidiana al nido. I contesti di cura, La Scuola, Brescia, 2007.

Montessori Maria, Manuale di pedagogia scientifica, Giunti, Firenze, 1970 (1912).

Mortari Luigina, La pratica dell’aver cura, Bruno Mondadori, Milano, 2006.

Muntoni Ludovica., I bambini pensano difficile. L'organizzazione delle idee nella scuola dell'infanzia, Carocci, Roma (2005).

Musi Elisabetta, Invisibili sapienze. Pratiche di cura al nido, Edizioni Junior-Spaggiari, Parma. 2011.

Pescioli Idana, La prima scuola, gli adulti all'opera con i bambini dai 3 ai 5 anni, Editori Riuniti, Roma, 1972.

Ritscher Penny, Slow School, Giunti, Firenze 2013.

Staccioli Gianfranco (con Ritscher Penny), Vivere a scuola. Programmare per situazioni, Carocci Editore, Roma, 2005.

Staccioli Gianfranco, Tra le righe, vivere volentieri la scuola di base, La Nuova Italia Editrice, Roma 1997.

Zavalloni Gianfranco, La pedagogia della lumaca, EMI, Bologna, 2009.

Agosto 2017.

Universidade do Estado de Santa Catarina - UDESC Programa de Pós-Graduação em Educação - PPGE 